\title{
Collagen gel droplet-embedded culture drug sensitivity testing in hard palate cancer-predicted antitumor efficacy of cetuximab: A case report
}

\author{
KANAME SAKUMA ${ }^{1}$, RYUKI TAMURA ${ }^{2}$, NAOTO NODA ${ }^{1}$, \\ MASUTAKA MIZUTANI $^{1}$, AKIRA YAMAGUCHI $^{1}$ and AKIRA TANAKA ${ }^{2}$ \\ ${ }^{1}$ Department of Oral and Maxillofacial Surgery, Niigata Hospital, The Nippon Dental University; ${ }^{2}$ Department of Oral \\ and Maxillofacial Surgery, The Nippon Dental University School of Life Dentistry at Niigata, Niigata 951-8580, Japan
}

Received March 28, 2017; Accepted July 28, 2017

DOI: $10.3892 / \mathrm{mco} .2017 .1367$

\begin{abstract}
In vitro anticancer drug sensitivity assessments have been performed for various types of cancer, and an association with clinical response has been observed. The collagen gel droplet-embedded culture drug sensitivity test (CD-DST) is an in vitro anticancer drug sensitivity test that has recently reported to be useful in oral squamous cell carcinoma (OSCC). CD-DST allows for the analysis of a smaller number of cells compared with other anticancer drug sensitivity tests. The present study reported a successful analysis of anticancer drug sensitivity using CD-DST on cervical lymph node tissue dissected following neoadjuvant chemotherapy from a 55-year-old man with advanced hard palate cancer. Tumor resection and bilateral neck dissection were performed following neoadjuvant chemotherapy (docetaxel + cisplatin + 5-fluorouracil; TPF) for hard palate cancer T2N2cM0. Local recurrence and cervical multiple skin metastasis occurred $\sim 8$ months after surgery, and the patient received six doses of cetuximab $(\mathrm{C}-\mathrm{mab})+$ cisplatin +5 -fluorouracil $(\mathrm{C}-\mathrm{mab}+\mathrm{PF})$ administration, which is a type of molecular-targeted therapy. Following the use of the CD-DST method, the clinical response was noted as stable disease following execution of TPF and partial response following execution of C-mab $+\mathrm{PF}$. In addition, low sensitivity by TPF and high sensitivity by $\mathrm{C}-\mathrm{mab}+\mathrm{PF}$ were reported. The CD-DST method reflected the clinical response for the patient, and the results of the current study indicate
\end{abstract}

Correspondence to: Kaname Sakuma, Department of Oral and Maxillofacial Surgery, Niigata Hospital, The Nippon Dental University, 1-8 Hamaura-cho, Chuo-ku, Niigata 951-8580, Japan E-mail: sakuma.k@ngt.ndu.ac.jp

Key words: anticancer drug sensitivity test, collagen gel droplet-embedded culture drug sensitivity test, oral squamous cell carcinoma, cetuximab, multiple drug combination that CD-DST is a useful tool for selecting chemotherapeutic drugs for patients with OSCC.

\section{Introduction}

The recent introduction of the anticancer drug cetuximab [an epidermal growth factor receptor (EGFr) inhibitor: C-mab] as a molecular-targeted therapy for treating head and neck cancers has expanded the scope of anticancer drugs for treating these cancers. However, no biomarkers are currently available that can predict $\mathrm{C}$-mab efficacy against oral squamous cell carcinoma (OSCC); therefore, it is typically administered irrespective of patient sensitivity (1). Hence, we investigated C-mab use with the collagen gel droplet-embedded culture drug sensitivity test (CD-DST). Kobayashi et al $(2,3)$ developed CD-DST which combines the collagen gel droplet culture method, a simple method of three-dimensional (3D) culture that allows a very small number of clinical samples to be tested with a serum-free medium step and quantitative evaluation by image analysis. CD-DST has little effect on non-cancerous cells, allowing accurate measurements of cancerous cells only. This method has been primarily used on tumors of the digestive system (4-6). Compared to such cancers of the primary organs, OSCC and other oral cancers generally have a smaller tumor volume. CD-DST is, therefore, likely to be a suitable method for testing the sensitivity of anticancer drugs on OSCC. However, there is little application of the CD-DST method to OSCC, with no evaluation of molecularly targeted drugs. In the present study, the CD-DST method was performed using a patient biopsy specimen of hard palate cancer to discern chemotherapy combined with a molecularly targeted drug at retrospective.

\section{Case report}

A 55-year-old man with hard palatal pain was referred to our institution in July 2012. An ulcerative mass measuring $35 \times 17 \times 8 \mathrm{~mm}^{3}$ with induration at the border of the hard palate was observed (Fig. 1). The patient also had bilateral neck metastasis at level IIa. The hard palatal mass was diagnosed as well-differentiated squamous cell carcinoma. 
Contrast-enhanced computed tomography (e-CT) showed a rim-enhanced mass at level IIa of the bilateral cervical area. In addition, it was close to the internal carotid artery (Fig. 2A). Positron emission tomography-computed tomography demonstrated high 18-fluorodeoxyglucose uptake at the hard palatal and cervical lymph nodes. The distant metastasis workup was negative. Diagnosis was hard palatal cancer, cervical lymph node metastasis (T2N2cM0: Stage IVA). CD-DST results revealed that the tumor was sensitive to various chemotherapeutic agents. The patients underwent one course of induction chemotherapy [docetaxel (DOC): $80 \mathrm{mg} /$ body; cisplatin (CDDP): $80 \mathrm{mg} /$ body; 5-fluorouracil (5-FU): 4,000 mg/body] following the diagnosis of hard palate cancer. The treatment effect of chemotherapy was confirmed with the Response Evaluation Criteria in Solid Tumors (RECIST) guideline version 1.1 (7). The size of the lymph node metastasis did not change as per e-CT after neoadjuvant chemotherapy. The therapy effect determination was stable disease (SD) (Fig. 2B). We performed maxillary malignant tumor resection and bilateral radical neck dissection under general anesthesia. However, left cervical lymph node metastasis recurrence and neck skin metastasis were observed eight months after surgery (Fig. 3). In accordance with the report of Vermorken et al (1), C-mab + CDDP + 5-FU (C-mab: 1450 mg/body; CDDP: $150 \mathrm{mg} /$ body; 5-FU: $5,000 \mathrm{mg} /$ body) was started and a total of six courses were performed. At the end of the six courses, we confirmed by CT that cervical lymph node recurrence and skin metastasis showed marked reduction of tumors, and therapy effect determination was partial response (PR) (Fig. 4). Thereafter, once weekly administration of C-mab alone was continued three times; however, there was a rapid increase in cervical skin metastatic tumors. The therapy effect determination became progressive disease (PD), and the treatment was canceled. As the symptoms worsened, it shifted to best support care focused on pain management. He died 330 days after cervical skin metastasis was confirmed.

The ethics committee of Nippon Dental University, School of Life Dentistry at Niigata (approval no. ECNG-H-119) approved study. CDDP, 5-FU, DOC, and C-mab, which are chemotherapeutic drugs frequently used for oral cancer, were assessed for in vitro chemosensitivity via the CD-DST method. CD-DST was performed according to the method described by Kobayashi et al $(2,3)$, using the Primaster ${ }^{\circledR}$ human cancer cell primary culture kit (Kurabo Industries Ltd., Osaka, Japan) (Fig. 5). Contact concentration and time of anticancer agents were as follows: $0.5 \mu \mathrm{g} / \mathrm{ml}$ CDDP (8), $0.7 \mu \mathrm{g} / \mathrm{ml} 5$-FU (8), $0.1 \mu \mathrm{g} / \mathrm{ml}$ DOC (9) contacted for $24 \mathrm{~h}$. The contact concentration of C-mab was set at $250 \mu \mathrm{g} / \mathrm{ml}$ for $144 \mathrm{~h}$, which is the maximum blood concentration during clinical administration. In addition, the test was also performed with a dose of $500 \mu \mathrm{g} / \mathrm{ml}$. Drug efficacy was determined via image analysis (Solution Systems Inc., Chiba, Japan). The growth rates of control incubations were calculated as the total volume on day $7 /$ total volume on day 0 . The in vitro sensitivity was expressed as the percentage $\mathrm{T} / \mathrm{C}$, where $\mathrm{T}$ was the total volume of the treated group and $\mathrm{C}$ was the total volume of the control group. When $\mathrm{T} / \mathrm{C}$ was less than or equal to $50 \%$, the in vitro drug sensitivity was regarded as effective. In contrast, when $\mathrm{T} / \mathrm{C}$ was greater than $50 \%$, sensitivity was considered as not effective (2,3). Clinical responses were
Table I. Results of drug sensitivity assessed by CD-DST and clinical response were compared.

\begin{tabular}{lcc}
\hline Drug & T/C & $\begin{array}{c}\text { Clinical } \\
\text { response }\end{array}$ \\
\hline CDDP & 97.6 & - \\
DOC & 76.9 & - \\
$5-F U$ & 83.2 & - \\
C-mab 250 $\mu \mathrm{g} / \mathrm{ml}$ & 65.5 & $\mathrm{PD}$ \\
$\mathrm{C}-\mathrm{mab} 500 \mu \mathrm{g} / \mathrm{ml}$ & 60.1 & \\
$\mathrm{CDDP}+5-\mathrm{FU}(\mathrm{PF})$ & 93.2 & - \\
$\mathrm{C}-\mathrm{mab}+\mathrm{CDDP}+5-\mathrm{FU}(\mathrm{C}-\mathrm{mab}+\mathrm{PF})$ & 32.4 & $\mathrm{PR}$ \\
$\mathrm{CDDP}+5-\mathrm{FU}+\mathrm{DOC}(\mathrm{TPF})$ & 71.6 & $\mathrm{SD}$ \\
\hline
\end{tabular}

In vitro drug sensitivity was defined as sensitive when the $\mathrm{T} / \mathrm{C}$ rate was $\leq 50 \%$. CDDP, Cisplatin; DOC, Docetaxel; 5-FU, 5-fluorouracil; C-mab, Cetuximab; PD, progressive disease; PR, partial response; $\mathrm{SD}$, stable disease; CD-DST, collagen gel droplet-embedded culture drug sensitivity test.

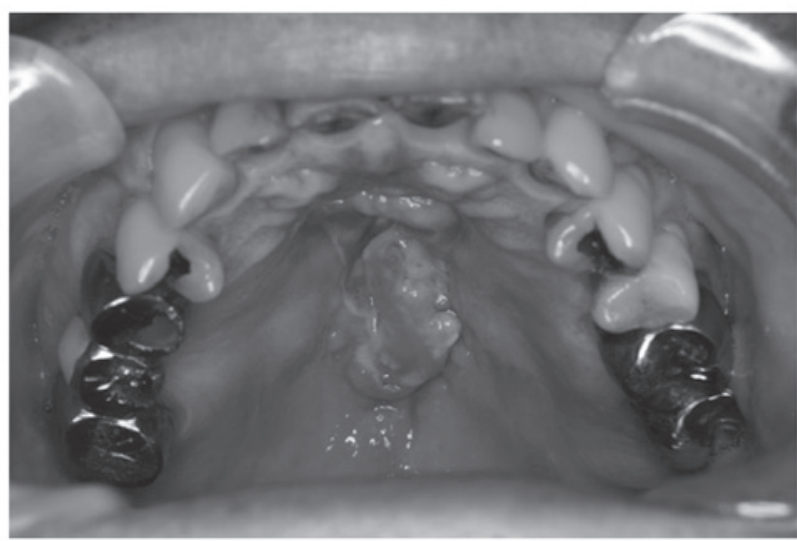

Figure 1. Clinical appearance at initial presentation revealed an ulcerative mass with induration, measuring $35 \times 17 \times 8 \mathrm{~mm}^{3}$, at the hard palate.

assessed according to RESIST, where tumors demonstrating a complete response (CR) or a PR were considered clinically responsive.

\section{Result}

Results of CD-DST and clinical response are shown in Table I. In CDDP single agent, T/C was $97.6 \%$, which was a low sensitivity. In DOC single agent, T/C was $76.9 \%$, which was a low sensitivity. In 5-FU single agent, T/C was $83.2 \%$, which was a low sensitivity. In C-mab $250 \mu \mathrm{g} / \mathrm{ml}$ single agent and C-mab $500 \mu \mathrm{g} / \mathrm{ml}$ single agent, T/C was 65.5 and $60.1 \%$, which was a low sensitivity. In multiple drug combination PF, T/C was $93.2 \%$, which was a low sensitivity. In C-mab + PF, $\mathrm{T} / \mathrm{C}$ was $32.4 \%$, which was a high sensitivity. In multiple drug combination TPF, T/C was $71.6 \%$, which was a low sensitivity. The clinical response of neoadjuvant chemotherapy (TPF) was SD. The clinical response of C-mab + PF was $\mathrm{PR}$. In addition, the clinical response of C-mab single agent was PD. 

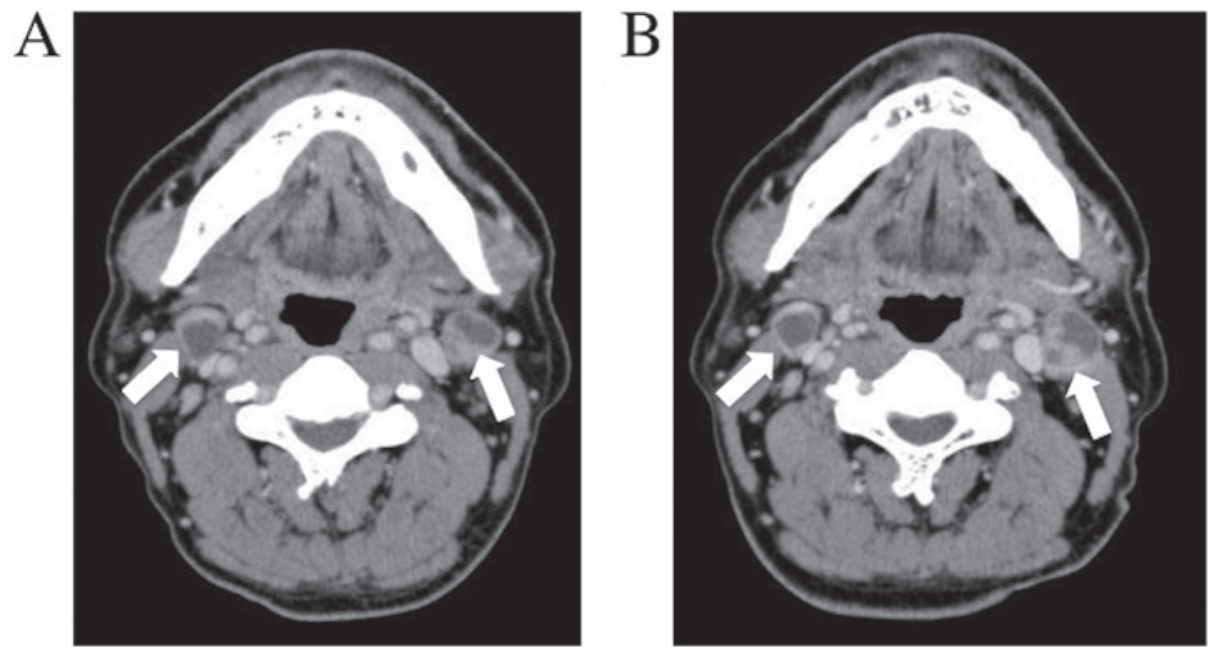

Figure 2. e-CT revealed a ring-enhanced mass at levels IIa of the bilateral cervical area at the first visit (A, arrows). In the e-CT evaluation after induction chemotherapy, there was no change in the size of cervical lymph node metastasis (B, arrows). E-CT, contrast-enhanced computed tomography.

\section{Discussion}

C-mab, which specifically binds to EGFr, is a promising novel chemotherapy drug for treating OSCC. However, there are no established predictors of OSCC therapeutic responses, and this inability to predict tumor response has resulted in drugs being administered to patients irrespective of tumor sensitivity. $\mathrm{C}$-mab reportedly causes infusion reactions and other serious adverse events $(1,10)$. Therefore, identifying a predictor of OSCC patient therapeutic response is essential to eliminating ineffective drug administration and associated patient risks. To further increase the effective rate of chemotherapy, an anticancer drug sensitivity test that accurately reflects the clinical prognosis is necessary.

Presently, various chemotherapy sensitivity tests have been developed and performed, such as HTCA, SDI, and HDRA (11-13). HDRA is an anticancer drug sensitivity test using 3D cell culture, but it entails problems such as the requirement for a large number of cells and the influence of contaminating fibroblasts. In contrast, CD-DST is capable of analyzing a small number of cells $\left(1 \times 10^{5}\right)$ in $3 \mathrm{D}$ cell cultures that create an environment close to that of the body and is unaffected by contaminating fibroblasts, thus achieving high positive and negative predictive values $(2,3)$.

Measurement success rates of $\geq 80 \%$ have been obtained for cancers, including colorectal cancer (14), lung cancer (15), and breast cancer (9), and a high clinical efficacy prediction rate of $91 \%$ has been obtained via CD-DST (3). This study evaluated its application to chemosensitivity testing for OSCC. In this study, it was possible to judge susceptibility to anticancer drugs without concerns such as the lack of cell numbers and bacterial contamination. OSCC and other oral cancers generally have a smaller tumor volume. Thus, CD-DST is likely to be a suitable method for testing the sensitivity of anticancer drugs on OSCC.

We performed CD-DST method on metastatic lymph node resected after neoadjuvant chemotherapy. In this study, the combination of TPF showed low sensitivity (T/C \%: 71.6). The effect of preoperative chemotherapy (TPF) was determined as
SD as lymph node metastasis did not change. In a retrospective examination, the CD-DST method and clinical response were consistent, suggesting that evaluation of multiple drug combination chemotherapy is possible in OSCC.

Furthermore, PF and C-mab each had low sensitivity with a single agent; however, when tested in combination, $\mathrm{T} / \mathrm{C}$ was $32.4 \%$, which was a high sensitivity value. The therapy effect determination was PR at the end of six cycles, consistent with the CD-DST method result. The CD-DST method could reproduce the synergistic effect of C-mab on $\mathrm{PF}$ therapy for OSCC. In this respect, synergistic antitumor activity has been reported in combination experiments with chemotherapeutic drugs or molecular-targeted therapeutic drugs. Combination treatment with cisplatin and gefitinib has confirmed in vitro sufficient additive inhibitory action on the survival of cancer cells (head and neck cancer) $(16,17)$. However, the putative mechanism through which $\mathrm{C}$-mab combination therapy enhances antitumor response remains to be demonstrated. Therefore, if the effect of PF $+\mathrm{C}-\mathrm{mab}$ can be evaluated by the CD-DST method, antitumor effect can be expected to be comprehensively judged without considering factors such as EGFr expression level and resistance gene. In addition, in this study CD-DST method, C-mab alone has a low sensitivity, but based on the EXTREME trial (1), we continued administering $\mathrm{C}$-mab alone once a week after six cycles of C-mab + PF. Cervical skin metastasis increased rapidly in about one month after changing to $\mathrm{C}$-mab single administration. The clinical response was $\mathrm{PD}$, and the susceptibility test of C-mab single agent was consistent with low sensitivity (C-mab $250 \mu \mathrm{g} / \mathrm{ml}: 65.5 \%, 500 \mu \mathrm{g} / \mathrm{ml}$ : $60.1 \%$ ). In this respect, our case report demonstrated that it was possible to evaluate even multi-drug-combined chemotherapy regimens, including C-mab. However, Anti-tumor effects of C-mab include antibody-dependent cell-mediated cytotoxicity (ADCC) activity and the like in addition to the signal inhibitory effect on EGFr (18), further study is necessary. The accumulation and analyses of additional cases are required to conduct a precise evaluation of CD-DST for patients with OSCC. 
A

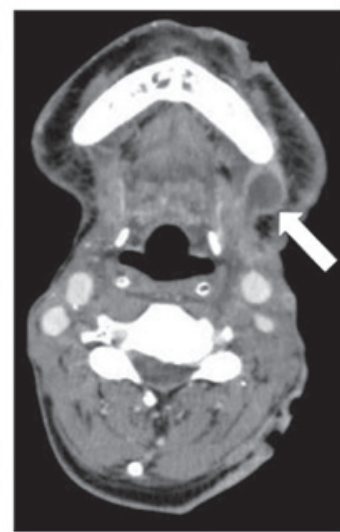

$\mathrm{B}$

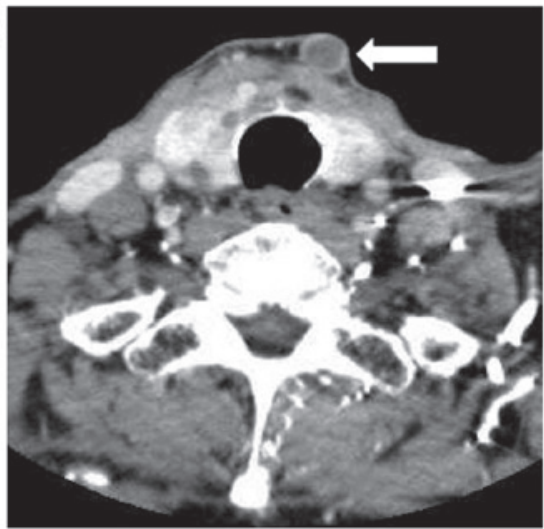

$\mathrm{C}$

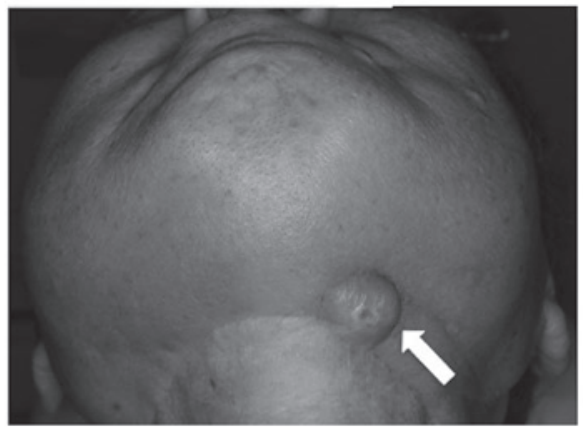

Figure 3. Contrast-enhanced computed tomography demonstrated a ring-enhanced mass at the skin of the cervical area observed at 1 year and 8 months after surgery (A and B, arrows). Left submandibular skin metastasis $\left(20 \times 18 \mathrm{~mm}^{2}\right)$ before administration of cisplatin +5 -fluorouracil + cetuximab $(\mathrm{C}$, arrow).
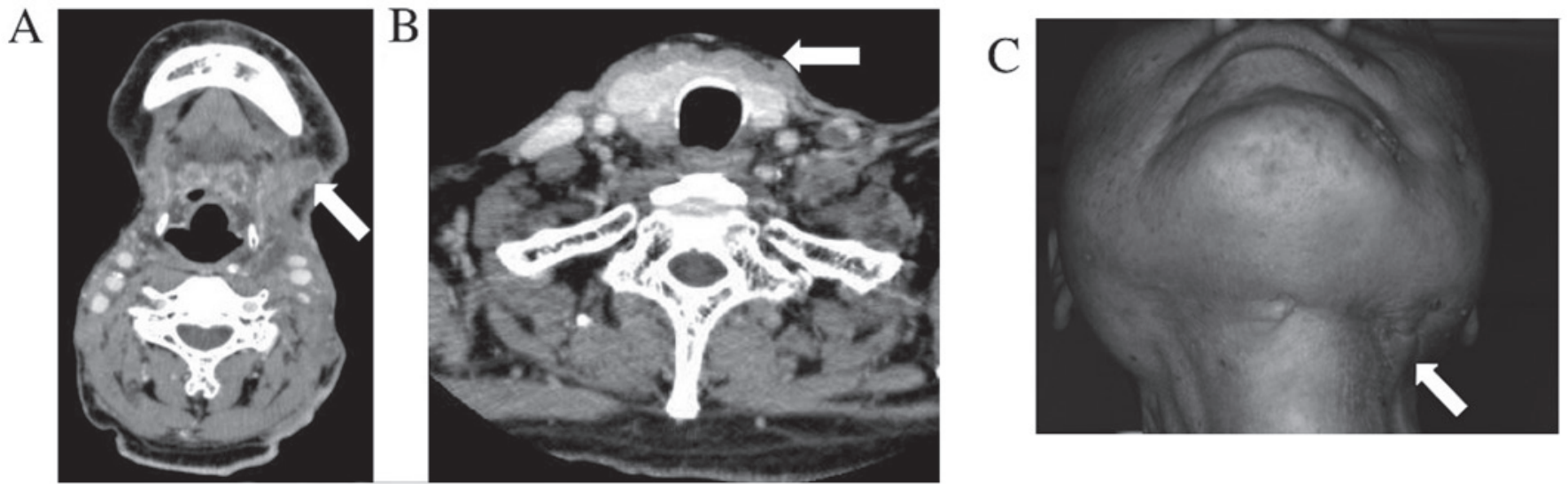

Figure 4. Skin metastasis demonstrated marked reduction in the tumor size, and therapy effect determination was partial response (A and B, arrows). Left submandibular skin metastasis became flat following administration of cisplatin + 5-fluorouracil + cetuximab (C, arrow).

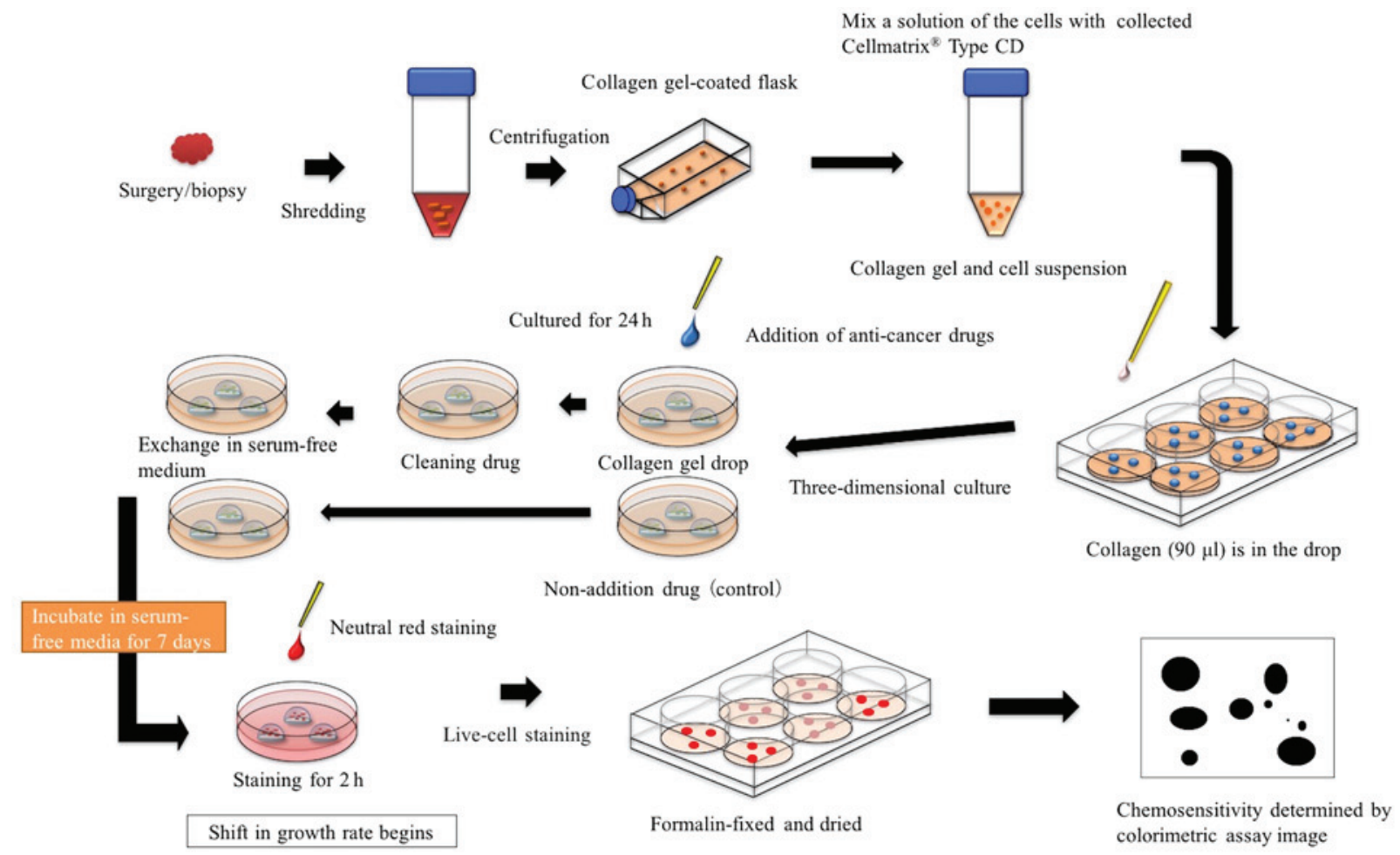

Figure 5. Overview of the collagen gel droplet-embedded culture drug sensitivity test method. 


\section{References}

1. Vermorken JB, Mesia R, Rivera F, Remenar E, Kawecki A, Rottey S, Erfan J, Zabolotnyy D, Kienzer HR, Cupissol D, et al: Platinum-based chemotherapy plus cetuximab in head and neck cancer. N Engl J Med 359: 1116-1127, 2008.

2. Kobayashi H, Tanisaka K, Kondo N, Mito Y, Koezuka M, Yokouchi H,Higashiyama M, Kodama K, Doi O, Yamada M, et al: Development of new in vitro chemosensitivity test using collagen gel droplet embedded culture and its clinical usefulness. Gan To Kagaku Ryoho 22: 1933-1939, 1995 (In Japanese).

3. Kobayashi H, Tanisaka K, Doi O, Kodama K, Higashiyama M, Nakagawa H, Miyake M, Taki T, Hara S, et al: An in vitro chemosensitivity test for solid human tumors using collagen gel droplet embedded cultures. Int J Oncol 11: 449-455, 1997.

4. Naitoh H, Yamamoto H, Murata S, Kobayashi H, Inoue K and Tani T: Stratified phase II trial to establish the usefulness of the collagen gel droplet embedded culture-drug sensitivity test (CD-DST) for advanced gastric cancer. Gastric Cancer 17: 630-637, 2014.

5. Ochiai T, Nishimura K, Watanabe T, Kitajima M, Nakatani A, Inou T, Washio M, Sakuyama N, Sato T, Kishine K, et al: Individualized chemotherapy for colorectal cancer based on the collagen gel droplet-embedded drug sensitivity test. Oncol Lett 4: 621-624, 2012 .

6. Mekata E, Sonoda H, Shimizu T, Tatsuta T, Yamaguchi T, Endo $\mathrm{Y}$ and Tani T: Clinical predictive value of in vitro anticancer drug sensitivity test for the therapeutic effect of adjuvant chemotherapy in patients with stage II-III colorectal cancer. Mol Clin Oncol 1: 763-767, 2013.

7. Eisenhauer EA, Therasse P, Bogaerts J, Schwartz LH, Sargent D, Ford R, Dancey J, Arbuck S, Gwyther S, Mooney M, et al: New response evaluation criteria in solid tumours: Revised RECIST guideline (version 1.1). Eur J Cancer 45: 228-247, 2009.

8. Sakuma K, Tanaka A and Mataga I: Collagen gel droplet-embedded culture drug sensitivity testing in squamous cell carcinoma cell lines derived from human oral cancers: Optimal contact concentrations of cisplatin and fluorouracil. Oncol Lett 12: 4643-4650, 2016.

9. Takamura Y, Kobayashi H, Taguchi T, Motomura K, Inaji H and Noguchi S: Prediction of chemotherapeutic response by collagen gel droplet embedded culture-drug sensitivity test in human breast cancers. Int J Cancer 98: 450-455, 2002.
10. Yoshino T, Hasegawa Y, Takahashi S, Monden N, Homma A, Okami K, Onozawa Y, Fujii M, Taguchi T, de Blas B, et al: Platinum-based chemotherapy plus cetuximab for the first-line treatment of Japanese patients with recurrent and/or metastatic squamous cell carcinoma of the head and neck: Results of a phase ii trial. Jpn J Clin 43: 524-531, 2013.

11. Salmon SE, Mamburger AW, Soehnlen B, Durie BG, Alberts DS and Moon TE: Quantitation of differential sensitivity of human tumor stem cells to anticancer drugs. N Engl J Med 298: 1321-1327, 1978.

12. Tanigawa N, Kern DH, Hisaka Y and Morton DL: Rapid assay for evaluating the chemosensitivity of human tumors in soft agar culture. Cancer Res 42: 2159-2164, 1982.

13. Kern DH, Dogemullar CR, Kennedy MC, Hildebrand-Zanki SU, Tanigawa N and Sondak VK: Development of miniaturized, improved nucleic acid precursor incorporation assay for chemosensitivity testing of human solid tumors. Cancer Res 45: 5436-5441, 1985

14. Araki Y, lsomoto H, Matsumoto A, Kaibara A, Yasunaga M, Hayashi K, Yatsugi H and Yamauchi K: An in vitro chemosensitivity test for colorectal cancer using collagen-gel droplet embedded cultures. Kurume Med J 46: 163-166, 1999.

15. Kawamura $M$, Inoue $Y$, Oyama $T$ and Kobayashi $K$ : Chemosensitivity test for unresectable non-small cell lung cancer: Nihon Geka Gakkai Zasshi 103: 229-232, 2002 (In Japanese).

16. Prewett M, Rockwell P, Rose C and Goldstein N: Anti-tumor and cell cycle responses in KB cells treated with a chimeric anti-EGFR monoclonal antibody in combination with cisplatin. Int J Oncol 9: 217-224, 1996.

17. Huang S, Armstrong EA, Benavente S, Chinnaiyan P and Harari PM: Dual-agent molecular targeting of the epidermal growth factor receptor (EGFR): Combining anti-EGFR antibody with tyrosine kinase inhibitor. Cancer Res 64: 5355-5362, 2004.

18. Kimura H, Sakai K, Arao T, Shimoyama T, Tamura T and Nishio K: Antibody- dependent cellular cytotoxicity of cetuximab against tumor cells with wild-type or mutant epidermal growth factor receptor. Cancer Sci 98: 1275-1280, 2007. 